\title{
Turnaround Time for Red Blood Cell Transfusion in the Hospitalized Patient: A Single-Center "Blood Ordering, Requisitioning, Blood Bank, Issue (of Blood), and Transfusion Delay" Study
}

\author{
Naveen Agnihotri, Ajju Agnihotri \\ Department of Blood Transfusion Medicine, Nayati Medicity, Mathura, Uttar Pradesh, India
}

\section{Abstract}

Background and Aim: The turnaround time (TAT) for blood transfusion (BT) is an important quality indicator for the health-care institutions undertaking this procedure. There is no established national or international benchmark for this TAT due to the dearth of a published literature. We thus studied the TAT and the contributory procedures leading to delay in commencing a red blood cell transfusion in the hospitalized patient. Materials and Methods: Delay was captured for the blood order transcription, requisitioning and sampling by the nurse, blood bank (BB) processing, blood issue, and the transfusion commencement in the hospitalized patients. The study was done prospectively over a 1-year period and involved all the patient locations spread over six floors in a tertiary care accredited hospital. Results: A total of 2022 blood requests were analyzed during the study period. Most (73\%) of the blood requests were marked as urgent by the treating unit. The average time from ordering to initiation of BT was $135 \mathrm{~min}$ in our study. BB processes (compatibility testing and issue) comprised approximately $47 \%$ of this delay $(63 \mathrm{~min})$, while rest of the delay happened in the processes (ordering $13 \mathrm{~min}$, sample transport $34 \mathrm{~min}$, and BT commencement $25 \mathrm{~min}$ ) outside the BB (72 min). Conclusion: Majority of the delay for blood transfusion happens due to the processes outside blood bank premises. Understanding the steps where delay happens has the potential to reduce the turnaround time for lifesaving procedures such as blood transfusion in the hospitalized patients.

Keywords: Blood arrangement, blood transfusion, delay, turnaround time

\section{INTRODUCTION}

Blood transfusion (BT) is one of the most common medical procedures performed in the modern hospitals. ${ }^{[1]}$ It is also perceived by the layperson as one of the most beneficial lifesaving interventions ${ }^{[2]}$ and possibly an indicator of the seriousness of the patient's ailment. Therefore, the time taken for starting a BT - the turnaround time (TAT) is an important quality indicator for the health-care institutions undertaking this procedure. A dearth of published literature on this important quality indicator is thus intriguing. In addition, there is no established national or international benchmark for the TAT for a BT. ${ }^{[3]}$ We thus decided to study the average time required to arrange a red blood cell (RBC) unit for transfusion in a hospitalized patient. We also aimed to determine the contribution of the individual processes - both

\begin{tabular}{|l|l|}
\hline \multicolumn{3}{|c|}{ Access this article online } \\
\hline Quick Response Code: & Website: \\
\hline & www.ijccm.org \\
\hline & \\
\hline
\end{tabular}

within and outside the blood bank (BB) which contribute to this timeline.

TAT can simply be defined as the period for completing a process cycle ${ }^{[4]}$ Clinicians and BB laboratory personnel may differ on the starting point for calculating this TAT. ${ }^{[5]}$ However, somewhat similar to a TAT for the laboratory report, the TAT for a BT should be calculated from the time a physician orders a $\mathrm{BT}$ to the time the patient starts receiving the $\mathrm{BT} .{ }^{[6]}$

Address for correspondence: Dr. Naveen Agnihotri, Department of Blood Transfusion Medicine, Nayati Medicity, NH-2, Mathura - 281 001, Uttar Pradesh, India. E-mail: naveenagnihotri@gmail.com

This is an open access journal, and articles are distributed under the terms of the Creative Commons Attribution-NonCommercial-ShareAlike 4.0 License, which allows others to remix, tweak, and build upon the work non-commercially, as long as appropriate credit is given and the new creations are licensed under the identical terms.

For reprints contact: reprints@medknow.com

How to cite this article: Agnihotri N, Agnihotri A. Turnaround time for red blood cell transfusion in the hospitalized patient: A single-center "Blood Ordering, Requisitioning, Blood Bank, Issue (of Blood), and Transfusion Delay" study. Indian J Crit Care Med 2018;22:825-30. 
Figure 1 outlines the typical steps (T1-T9) involved in a BT to a hospitalized patient. Minimum TAT in starting a BT to a patient in any setup is the collective time taken for all these steps. BT process starts from the patient (the treating doctor orders the BT) and again ends at the patient (BT), thus completing an orbit. The acronym "ORBIT" also stands for the principal measurable and time-consuming steps in a typical BT process - Ordering, Requisitioning, BB, Issue (of blood), and Transfusion initiation.

\section{Materials and Methods}

The study was conducted over a 1-year period (November 2016-October 2017) in a tertiary care hospital-based BB in a Tier III city in a northern state of India. The study was done only for the packed red blood corpuscle transfusions as this is the most common and also the most time-consuming blood component required for the patient transfusion.

The BB was located on the first floor of the six-floor hospital building which has multiple lifts and stairs for swift movement between the floors. Clinically stable patients were kept in the wards/rooms situated on the various floors of the hospital building. Unlike intensive care units, wards were not clinical specialty specific and were allotted on the basis of the availability and the financial preference of the patient. An informed consent for the BT was required only for the $1^{\text {st }}$ transfusion and was valid for the duration of that particular hospital admission. Whenever the treating doctor decided to transfuse the blood, it was documented in the patient case file [Figure 1-T1] for the nurse to execute the request. No verbal orders were allowed as per the hospital policy. The blood ordering and the blood sample label [Figure 1-T2] generation happened through the (computerized) hospital information system (HIS). The blood sample when received in the BB [Figure 1-T4] was acknowledged in the same HIS by the BB staff on duty. Therefore, the blood sample draw time and the receiving time were captured digitally and on a real-time basis. Time taken for the sample transport from the patient bedside to the BB [Figure 1-T3] was indirectly calculated from

\footnotetext{
(T1) Treating doctor orders a blood transfusion for the patient $\downarrow$

(T2) Requisition form filled; consent and blood sample(s) taken $\downarrow$

(T3) Sample transport arranged (relative/attendant/ pneumatic tube) $\downarrow$

(T4) Sample received in blood bank $\downarrow$

(T5) Compatibility testing and labeling in blood bank $\downarrow$

(T6) Demand from ward to issue blood for transfusion $\downarrow$

(T7) Blood unit carried from the blood bank (relative/ attendant/pneumatic tube)

(T8) Blood received at patient location and documents verified $\downarrow$

(T9) Blood Transfusion initiated to the patient
}

Figure 1: Steps in arranging a blood transfusion - the blood transfusion orbit
T4 (blood sample received) and T2 (blood sample labeled) as this time (T3) could not have been calculated directly. All other data, i.e., from the compatibility testing in the BB to the start of BT [Figure 1-T5-T9] were obtained from the appropriate records where the concerned health-care worker (HCW) captured the data on a real-time basis.

Time when the treating doctor prescribed for a BT (T1) was captured from the patient file. Since this time (T1) was not available in the HIS or the BB records, it was obtained from a random audit of $10 \%$ of the patient files. Accordingly, patient records were audited for whom a BT was ordered as well as transfused during the study period. These samples were randomly taken from all the patient locations. To correctly capture this time (T1), a senior technician was assigned to scrutinize the patient records along with the nurse at the patient location. This activity (of capturing the time T1) was spread over the study period to avoid any bias due to the change in the doctor's roster in the wards.

Blood compatibility testing (T5) in the BB included blood grouping, irregular antibody screening, and indirect antiglobulin phase crossmatch (collectively called as serological testing) for all the blood units included in the study. All the cases where irregular antibodies in the patient serum were detected and which required further detailed investigations were excluded from the study. Both automated and semi-automated systems based on the column agglutination technology were used for the serological testing. The treating doctor had to select the urgency of the BT from the three categories mentioned on the requisition form - immediate (within $7 \mathrm{~min}$ and pending complete compatibility testing), urgent (within $55 \mathrm{~min}$ ), and routine $(8-10 \mathrm{~h})$.

Every time the blood unit(s) was issued (T6) from the BB, it was termed as a blood issue episode for the study purpose. Therefore, one blood request for a particular patient would translate into one or more blood issue episodes, depending on the patient requirement at a given time.

BT start time (T9) was noted by the nurse in the appropriate column on the blood compatibility report issued by the BB. This compatibility report was mandatorily returned within a period of $24-48 \mathrm{~h}$ by the nurse to the BB. The timeline for starting the BT was calculated from the difference between the $\mathrm{T} 9$ and $\mathrm{T} 6$ (issue time from the $\mathrm{BB}$ ).

ORBIT delays were calculated as follows [Figure 1]:

1. Ordering TAT: $\mathrm{T} 2-\mathrm{T} 1$

2. Requisitioning TAT: $\mathrm{T} 4-\mathrm{T} 2$

3. BB TAT: $\mathrm{T} 5-\mathrm{T} 4$

4. Issue TAT: T7 - T6

5. Transfusion start TAT: T9 - T7.

The time when the unit was kept arranged in the BB (T6-T5) was excluded from the study as this was not a delay in arranging the blood. Rather, this was due to the patient-related factors, for example, a patient posted for routine surgery the next day, etc. 


\section{Statistical analysis}

Since the distribution of data is non-Gaussian so nonparametric value - median was taken into the consideration. Median time was calculated for the different wards and intensive care units. Time consumed for BB compatibility testing was predictable, and therefore, an average was calculated rather than the median.

\section{RESULTS}

\section{Ordering turnaround time}

A median timeline of $13 \mathrm{~min}$ (range: 3-32 min) was noted in translating the doctor's blood order by the nurse at various patient locations [Table 1].

\section{Requisitioning turnaround time}

Various types of wards/intensive care units and their respective physical distance from the BB are given in Table 2 . The median requisitioning TAT, i.e., the time taken for the signed blood request along with the patient's blood sample(s) to reach the $\mathrm{BB}$ reception was found to be $34 \mathrm{~min}$ [Table 2].

\section{Blood bank turnaround time}

Out of a total of 2022 compatibility tests done during the study period, majority of the blood requests were labeled as urgent by the treating unit [Table 3]. Not considering the urgency of the blood request, a median TAT of 55 min was noted for the compatibility testing, done in the BB.

\section{Issue turnaround time}

The time taken to confirm the patient details on the blood unit, prepare compatibility documents, and make arrangement for the blood unit transport and a final check on the blood unit took an average of $8 \mathrm{~min}$ in the BB. This timeline was calculated for a total of 2039 blood issue episodes.

\section{Transfusion turnaround time}

After release of the blood unit from the BB, the median TAT for starting the BT to the patient was found to be $25 \mathrm{~min}$ for a total of 2039 blood issue episodes [Table 4].

\section{Cumulative Ordering, Requisitioning, Blood Bank, Issue (of blood), and Transfusion delay}

Table 4 shows the ward-wise TAT for starting a BT to an admitted patient in the hospital. An average timeline of $135 \mathrm{~min}$ (range: 56-162 $\mathrm{min}$ ) from the doctor's order to the start of BT was found in our study.

\section{DISCUSSION}

Timely BT to a patient is a lifesaving procedure; however, average delay before a BT can be started to a hospitalized patient in an Indian setup has not been studied in the past. A typical BT process in India is slightly different from that practiced in most of the developed nations. The treating doctor recommends a BT, and the nurse translates this order into a signed blood requisition. The nurse then obtains the patient's blood samples which are then carried to the BB. BB staff processes this blood

\begin{tabular}{lcc}
\hline $\begin{array}{l}\text { Table 1: Delay in translating the treating doctor's order } \\
\text { by the nurse (ordering delay) }\end{array}$ & $\begin{array}{c}\text { Number of files } \\
\text { scrutinized }\end{array}$ & $\begin{array}{c}\text { Delay } \\
\text { (min) }\end{array}$ \\
\hline Location & 26 & 11 \\
& 44 & 16 \\
\hline ER & 14 & 32 \\
$1^{\text {st }}$ floor (general ward) & 7 & 3 \\
CCU/CTVS ICU/Cath lab & 32 & 32 \\
LDR & 5 & 3 \\
Surgical ICU & 19 & 7 \\
Dialysis & 16 & 10 \\
HDU & 21 & 10 \\
Medical ICU & $1^{*}$ & 10 \\
Pediatric/neonatal ICU & 19 & 6 \\
$4^{\text {th }}$ floor (single/double patient rooms) & 203 & 13 \\
$6^{\text {th }}$ floor (single/double patient rooms) & Grand total & \\
*Very few blood requests were received from this particular floor; \\
value for this floor was thus ignored in calculating the overall average \\
delay (13 min). CCU: Critical care unit; CTVS: Cardiothoracic \\
vascular surgery; ICU: Intensive care unit; Cath lab: Catheterization \\
laboratory; ER: Emergency room; LDR: Labor and delivery room; \\
HDU: High-dependency unit
\end{tabular}

\begin{tabular}{|c|c|c|c|}
\hline Location & $\begin{array}{c}\text { Distance } \\
\text { from the BB } \\
\text { (floors)* }^{*}\end{array}$ & $\begin{array}{l}\text { Number of } \\
\text { requests }\end{array}$ & $\begin{array}{l}\text { Delay } \\
\text { (min) }\end{array}$ \\
\hline ER & -1 & 388 & 27 \\
\hline $1^{\text {st }}$ floor (general ward) & 0 & 396 & 48 \\
\hline $\mathrm{CCU} / \mathrm{CTVS}$ ICU/Cath lab & +1 & 146 & 27 \\
\hline LDR & +1 & 78 & 33 \\
\hline Surgical ICU & +1 & 280 & 30 \\
\hline Dialysis & +2 & 41 & 32 \\
\hline $\mathrm{HDU}$ & +2 & 197 & 29 \\
\hline Medical ICU & +2 & 225 & 40 \\
\hline Pediatric/neonatal ICU & +2 & 80 & 39 \\
\hline $4^{\text {th }}$ floor (single/double patient rooms) & +3 & 32 & 22 \\
\hline $6^{\text {th }}$ floor (single/double patient rooms) & +5 & 159 & 53 \\
\hline Grand total & & 2022 & 34 \\
\hline \multicolumn{4}{|c|}{$\begin{array}{l}\text { *Minus sign means at a lower floor and plus indicates number of } \\
\text { floors above the BB; BB was located on the } 1^{\text {st }} \text { floor of the hospital } \\
\text { building. ER: Emergency room; BB: Blood bank; CCU: Critical care } \\
\text { unit; CTVS: Cardiothoracic vascular surgery; ICU: Intensive care unit; } \\
\text { Cath lab: Catheterization laboratory; ER: Emergency room; LDR: Labor } \\
\text { and delivery room; HDU: High-dependency unit }\end{array}$} \\
\hline
\end{tabular}

request and performs the compatibility testing and issues the blood unit for the transfusion. The relative/HCW/pneumatic chute carries this blood unit to the nurse/doctor who then tallies the information and checks the blood before finally starting a BT. Therefore, a typical BT process commences from a patient's blood order, passes through a series of steps involving multiple $\mathrm{HCW}$, and again ends at a point when a BT is started to the patient, thus completing a full "orbit."

We planned this Blood ORBIT study to calculate the time consumed in arranging a BT for a patient in an average Indian hospital setup with an in-house BB. 
It is a general belief among the clinical teams that there is usually an unknown delay in arranging blood from the BB. This general belief is clearly reflected in the fact that majority $(73 \%)$ of the blood requests in our study were marked as "urgent." It is generally felt that marking blood requests as "urgent" would cut short the delay in arranging the blood, to the minimum. This belief probably also reflects the uncertainty in treating team's mind, about the exact time in arranging a BT for a patient. In health-care systems with more mature and predictable processes, a far lesser number of blood requests are anticipated to be marked as urgent. A prospective study in the North of England found that only $25 \%$ of the blood demands were marked as urgent (required within $1 \mathrm{~h}$ ). ${ }^{[7]}$

At the commencement of the BT process, a TAT of $13 \mathrm{~min}$ in translating the doctor's BT order by the nurse was an interesting finding of our study. This "ordering" timeline varied from 3 to $32 \mathrm{~min}$ [Table 1] and reflects the obvious variability in the different patient locations. Surgical intensive care unit showed the highest TAT ( $32 \mathrm{~min}$ ) for the blood order translation by the nurse. This delay is most likely due to the multitude of blood samplings, dressings, and miscellaneous work required of the nurses in such locations. Ordering TAT constitutes $10 \%$ of the total delay [Figure 2] in arranging a BT and can be further improved as highlighted by a recent review study on the nurse-physician communication. ${ }^{[8]}$ The use of technology by the treating clinicians for blood ordering may obviate the need

\begin{tabular}{lcc}
\hline Table 3: Blood bank delay \\
\hline $\begin{array}{l}\text { Type of } \\
\text { blood } \\
\text { request }\end{array}$ & $\begin{array}{c}\text { Number of requests } \\
\text { (percentage of } \\
\text { total) }\end{array}$ & $\begin{array}{c}\text { Average delay in } \\
\text { minutes for the } \\
\text { compatibility testing }\end{array}$ \\
\hline Urgent & $1474(72.9)$ & 52 \\
Routine & $514(25.4)$ & 60 \\
Immediate* & $34(1.7)$ & 40 \\
Total & $2022(100.0)$ & 55 \\
\hline
\end{tabular}

*Blood was issued within 7-min pending compatibility testing, which in turn was completed on a priority basis even after the blood issue for this step and thus this delay. However, as a prerequisite for this technological intervention, blood banking would have to graduate to the policy of "type and screen" from the current "type and crossmatch"[9] as discussed later.

An unexpectedly huge delay of 34 min was noted in transporting the blood sample from the patient location to the BB. This delay happens mainly because of two reasons: first - arranging for a courier (a hospital staff as in our study or the patient's relative in some other hospitals) and second - the physical transit time from the patient location to the BB. This "requisitioning" TAT had no prima facie relationship with the physical distance between the patient and the BB [Table 2] and was thus not explored further. Hence, the main cause of this requisitioning delay was the time lost in arranging for a courier. In our study, the hospital staff used as a courier was a multipurpose worker, involved in miscellaneous works, such as patient transport, patient cleaning, and medication arrangement from the pharmacy. The nonavailability of a courier at a particular point of time for the blood sample transport is the most plausible reason for this requisitioning delay and thus needs to be studied further. A similar finding of delayed

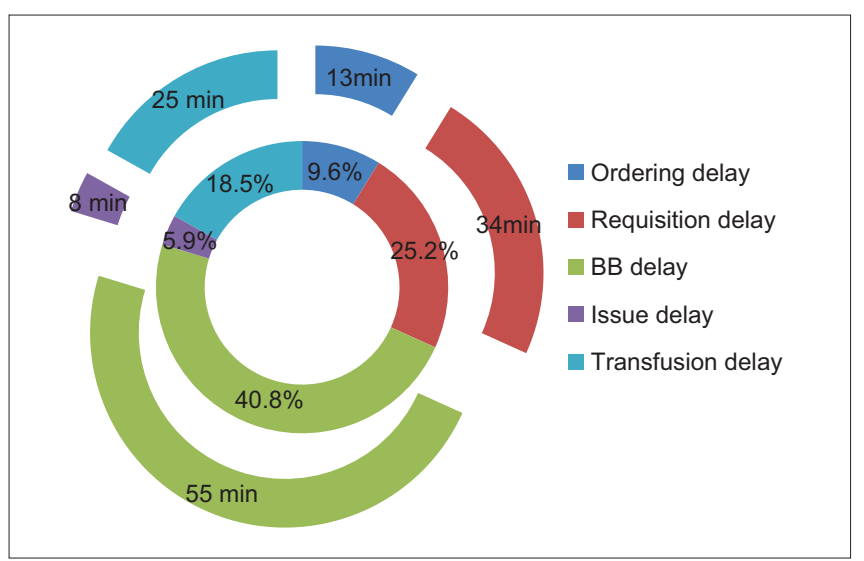

Figure 2: Cumulative Ordering, Requisitioning, Blood Bank, Issue (of Blood), and Transfusion delay in arranging a blood transfusion

\begin{tabular}{|c|c|c|c|c|c|c|c|}
\hline Location & $\begin{array}{l}\text { Distance from the } \\
\text { BB (floor wise) }\end{array}$ & $\begin{array}{l}\text { Ordering } \\
\text { delay (min) }\end{array}$ & $\begin{array}{l}\text { Requisition } \\
\text { delay (min) }\end{array}$ & $\begin{array}{l}\text { BB delay } \\
\text { (min) }\end{array}$ & $\begin{array}{l}\text { Issue delay } \\
\text { (min) }\end{array}$ & $\begin{array}{c}\text { Transfusion } \\
\text { start delay (min) }\end{array}$ & $\begin{array}{l}\text { Total delay } \\
\text { (min) }\end{array}$ \\
\hline ER & -1 & 11 & 27 & $00^{*}$ & 8 & 10 & 56 \\
\hline $1^{\text {st }}$ floor (general ward) & 0 & 16 & 48 & 55 & 8 & 30 & 157 \\
\hline CCU/CTVS ICU/Cath lab & +1 & 32 & 27 & 55 & 8 & 20 & 142 \\
\hline LDR & +1 & 3 & 33 & 55 & 8 & 40 & 139 \\
\hline Surgical ICU & +1 & 32 & 30 & 55 & 8 & 25 & 150 \\
\hline Dialysis & +2 & 3 & 32 & 55 & 8 & 15 & 113 \\
\hline HDU & +2 & 7 & 29 & 55 & 8 & 25 & 124 \\
\hline Medical ICU & +2 & 10 & 40 & 55 & 8 & 20 & 133 \\
\hline Pediatric/neonatal ICU & +2 & 10 & 39 & 55 & 8 & 45 & 157 \\
\hline $4^{\text {th }}$ floor (single/double patient rooms) & +3 & 10 & 22 & 55 & 8 & 33 & 128 \\
\hline $6^{\text {th }}$ floor (single/double patient rooms) & +5 & 6 & 53 & 55 & 8 & 40 & 162 \\
\hline Overall median & NA & 13 & 34 & 55 & 8 & 25 & 135 \\
\hline
\end{tabular}

*Blood was issued immediately pending compatibility testing hence 0 min. CCU: Critical care unit; CTVS: Cardiothoracic vascular surgery; ICU: Intensive care unit; Cath lab: Catheterization laboratory; ER: Emergency room; LDR: Labor and delivery room; HDU: High-dependency unit 
courier arrival leading to the prolonged TAT for RBC issue was observed in a recent study by a South Korean center. ${ }^{[3]}$

This requisitioning TAT contributes $25 \%$ to the total delay in arranging a BT [Figure 2]. It can be improved by the use of technology like pneumatic chute system ${ }^{[10]}$ or by having dedicated personnel for such transports. However, studies on cost-benefit analysis would be required before such an implementation is actually attempted. Nonetheless, any major decrease in the requisition TAT has the potential to cut short the overall delay in arranging a BT.

The average time taken by the BB to make the blood units available in our study was $55 \mathrm{~min}$. This comprised blood grouping, irregular antibody screening, compatibility testing using Coombs phase technique, and mandatory documentation with labeling. This testing policy is commonly referred to as "type and crossmatch" policy (TXm policy). The processing time taken by any BB is dependent on the extent of automation, type of analyzer used, experience of the staff, workload, etc. ${ }^{[11,12]}$ The time taken for this step in our study is similar to that found by Aggarwal et al. in their recent study from North India (79 min) using almost the same equipment and processes as used in our study. ${ }^{[9]}$ The TAT in BB constitutes nearly $41 \%$ of the total delay [Figure 2] in arranging a BT for a patient. Hence, measures which can decrease this BB timeline can have a major impact on the overall TAT for arranging a BT.

As against TXm policy used in our center and most of the $\mathrm{BB}$ in India, blood center in developed countries uses "Type and Screen" policy (TS policy). Center using this TS policy has reported an average time from $26^{[13]}$ to $33 \mathrm{~min}^{[14]}$ for the processing by the BB. A study involving 466 institutions reported this $\mathrm{BB}$ processing time to be between 30 and $35 \mathrm{~min} .{ }^{[13]}$ The main reason for the big time difference in our study (55 $\mathrm{min})$ versus these centers $(26-35 \mathrm{~min}$ ) is the difference in the compatibility policy (TXm vs. TS policy). The policy of a precompleted serological testing before an actual blood requirement is called as "Type and Screen" policy (TS policy) for compatibility testing. In this policy, any patient anticipated to receive a BT is typed for $\mathrm{ABO} / \mathrm{Rh}$ and screened for any irregular antibody, even before a blood request is raised. Therefore, under this policy, whenever a BT is required, just an abbreviated crossmatch is required before a blood unit can be released. This TS policy thus decreases the processing time in BB when an actual BT is needed. ${ }^{[7]}$ Showing the advantages of TS policy vis-à-vis TXm policy, Aggarwal et al. demonstrated that the TAT could be significantly reduced by such an implementation in the Indian context also. ${ }^{[9]}$ Therefore, an informed decision by the hospital administration to implement the TS policy for blood compatibility testing can significantly reduce the overall TAT. ${ }^{[13]}$

Once a blood unit is arranged and before it can be actually released from the $\mathrm{BB}$, there is an "issue" delay. The 8-min timeline for a blood issue was comparable with the other studies, which found an issue TAT of 6-21 min. ${ }^{[13,15]}$ The authors believe that due to the gravity of the task (final check before release), there is hardly any scope for further reducing this time. Irrespective of whether a manual or an automated system was used, the issue time has averaged around 5-7 min in most of the studies. ${ }^{[13,15]}$

The TAT for starting the BT to the patient after the issue from the BB was called as "transfusion start" delay. This represented the timeline in transporting the unit to the patient bedside by the courier, checking and tallying of the patient-blood unit details, and preparing the patient for the transfusion. This TAT of $25 \mathrm{~min}$, which constitutes nearly $19 \%$ to the total delay, may be because of the involvement of multiple $\mathrm{HCW}$ in this final step before a BT was started to a patient.

\section{Stand-alone blood banks}

While our study was conducted in a hospital-based BB, it is pertinent to discuss the presumed ORBIT delay in the stand-alone BBs (SABBs). This category of BB constitutes nearly $14 \%-35 \%$ of the total BBs in India ${ }^{[16]}$ and is typically situated out of the hospital premises. The requisitioning delay as well as the transfusion start delay (transporting sample to the $\mathrm{BB}$ and blood unit from the BB, i.e., $\mathrm{R}$ and $\mathrm{T}$ of the ORBIT) can be presumed to be much more than for an in-house BB. Similarly, due to the remote location of the patient, the BB staff is not under direct pressure to release the unit in the shortest possible time. This can make the BB and issue delay (B and I of the ORBIT) on the higher side as compared to our study. Therefore, "RBIT" of the ORBIT delay can be presumed to be even higher than found in our study. Nonetheless, in the absence of any study on ORBIT delay in such SABB, such a presumption can best be called an educated guess.

\section{Total time for blood delivery [Figure 2]}

The median time from request to retrieval of blood component at the patient bedside is $39 \mathrm{~min}$ in the Western countries ${ }^{[13]}$ as compared to $135 \mathrm{~min}$ in our study. Different category of patients may be affected differently due to this ORBIT delay. For example, those requiring blood in an emergency are impacted by the entire ORBIT delay while those undergoing routine surgeries are more affected by the "IT" of the ORBIT delay. Thus, depending on the major category of the patients catered, each institution can prioritize the focused study and/ or improvement of either the entire ORBIT (delay) or just the "IT" of the ORBIT (delay).

\section{Limitations of the study}

Since the main objective of our study was to find the average timeline and crucial procedures contributing to it, the exact factors and their respective contribution to the delay were not studied. These factors may vary across locations and need to be studied further. For example, center using an electronic blood ordering system and pneumatic chute is expected to have shorter "ordering" and "requisitioning" timelines, respectively, as compared to our study. Nonetheless, our center represents the scenario and processes used in majority of the Indian BBs and hospitals. Therefore, the finding of our study still serves as 
a crucial stepping stone for benchmarking this critical quality indicator - TAT for a BT.

\section{Conclusion}

Of the total time consumed in arranging a red blood cell transfusion for a hospitalized patient, more than half is due to the processes happening outside the blood center. A turnaround time of $135 \mathrm{~min}$ for starting a blood transfusion seems unreasonably long. Policy revisions for the blood/sample transport and the compatibility testing in the blood bank have the potential to reduce this timeline to nearly one-third of the current delay. More studies in different health-care settings are required to highlight more measures for decreasing the turnaround time for a blood transfusion in a hospitalized patient in India.

\section{Financial support and sponsorship}

Nil.

\section{Conflicts of interest}

There are no conflicts of interest.

\section{RefERENCES}

1. Pfuntner A, Wier LM, Stocks C. Most Frequent Procedures Performed in U.S. Hospitals, 2010. HCUP Statistical Brief \#149. Rockville, MD: Agency for Healthcare Research and Quality; February, 2013. Available from: http://www.hcup-us.ahrq.gov/reports/statbriefs/sb149.pdf. [Last accessed on 2018 Aug 28].

2. Ngo LT, Bruhn R, Custer B. Risk perception and its role in attitudes toward blood transfusion: A qualitative systematic review. Transfus Med Rev 2013;27:119-28.

3. Lee AJ, Kim SG. Analysis of turnaround time for intraoperative red blood cell issues: A single-center study. Lab Med 2017;48:277-81.
4. Available from: http://www.businessdictionary.com/definition/ turnaround-time. [Last accessed on 2018 Apr 26].

5. Howanitz PJ, Cembrowski GS, Steindel SJ, Long TA. Physician goals and laboratory test turnaround times. A college of American pathologists Q-probes study of 2763 clinicians and 722 institutions. Arch Pathol Lab Med 1993;117:22-8.

6. Pati HP, Singh G. Turnaround time (TAT): Difference in concept for laboratory and clinician. Indian J Hematol Blood Transfus 2014;30:81-4.

7. Tinegate HN, Davies T, Elshaw RJ, Jane G, Lyon M, Norfolk DR, et al. When and why is blood crossmatched? A prospective survey of transfusion laboratory practice in two regions in the North of England. Vox Sang 2010;99:163-7.

8. Tan TC, Zhou H, Kelly M. Nurse-physician communication - An integrated review. J Clin Nurs 2017;26:3974-89.

9. Aggarwal G, Tiwari AK, Arora D, Dara RC, Acharya DP, Bhardwaj G, et al. Advantages of type and screen policy: Perspective from a developing country! Asian J Transfus Sci 2018;12:42-5.

10. Pupek A, Matthewson B, Whitman E, Fullarton R, Chen Y. Comparison of pneumatic tube system with manual transport for routine chemistry, hematology, coagulation and blood gas tests. Clin Chem Lab Med 2017;55:1537-44.

11. Sackett K, Kjell A, Schneider AM, Cohn CS. A field analysis trial comparing the turnaround times of routine and STAT red blood cell immunohematology testing. Immunohematology 2017;33:1-5.

12. Bajpai M, Kaur R, Gupta E. Automation in immunohematology. Asian J Transfus Sci 2012;6:140-4.

13. Novis DA, Friedberg RC, Renner SW, Meier FA, Walsh MK. Operating room blood delivery turnaround time: A college of American pathologists Q-probe study of 12647 units of blood components in 466 institutions. Arch Pathol Lab Med 2002;126:909-14.

14. Kim SH, Chung YN, Kim YR, Kang SH. Analysis of turnaround times of blood issue at an Island hospital. Korean J Blood Transfus 2012;23:217-23.

15. McClain CM, Hughes J, Andrews JC, Blackburn J, Sephel S, France D, et al. Blood ordering from the operating room: Turnaround time as a quality indicator. Transfusion 2013;53:41-8.

16. Choudhury N. Need to change present regulatory framework for blood banks in India. Asian J Transfus Sci 2011;5:1-2. 